\title{
Hiperglucemia como factor pronóstico de mortalidad en pacientes con SARS-CoV-2
}

\author{
Hyperglycemia as a prognostic factor of \\ mortality in patients with SARS-CoV-2 \\ Luis Arturo Camacho-Saavedra', \\ Andy Grover Zavaleta-Carranza², \\ Daniel Octavio Trigoso-Aranda²
}

\author{
Camacho-Saavedra L, Zavaleta-Carranza A, Trigoso-Aranda D. \\ Hiperglucemia como factor pronóstico de mortalidad en pacientes con \\ SARS-CoV-2. Rev Soc Peru Med Interna. 2020;33(4):I5I-I 54. \\ https://doi.org/10.36393/spmi.v33i4.562
}

\begin{abstract}
RESUMEN
Se investigó si la hiperglucemia es un factor pronóstico de mortalidad en pacientes infectados con SARS-Cov 2. Se buscó artículos en las bases de datos de PubMed y Google Scholar hasta el 30 de Julio del 2020. Se utilizó las palabras claves: " Hiperglucemia “," Síndrome Respiratorio Agudo Grave “, “ Neumonía Viral “, " Virosis “, “" Infecciones por Coronavirus “, " período de incubación ",” transmisión "," características clínicas “, "fisiopatología” , "hiperglucemia”, con la interposición del operador booleano” Y “"”. Además, accedimos a la literatura científica y las recomendaciones actualmente disponibles en los sitios web de la OMS y los Centros para el Control y la Prevención de Enfermedades (CDC) de los Estados Unidos. Los pacientes infectados por SARS-CoV-2 y con hiperglucemia (glucemia >I $80 \mathrm{mg} / \mathrm{dL}$ ) presentaron una mayor mortalidad en comparación con pacientes con una glucemia controlada. La hiperglucemia aguda o crónica y, más aún, cuando permanece no controlada se asocia con un aumento de la gravedad y la mortalidad en pacientes infectados con SARS-CoV-2.
\end{abstract}

Palabras clave: Hiperglucemia, síndrome respiratorio agudo grave, neumonía viral, viral, infecciones por coronavirus, COVID-I9.

\section{ABSTRACT}

A research was made on whether hyperglycemia is a predictor of mortality in patients infected with SARS-Cov 2. Articles were searched in the PubMed and Google Scholar databases until July 30, 2020. The keywords were used: "Hyperglycemia", "Serious Acute Respiratory Syndrome", "Viral Pneumonia", "Virosis", "Coronavirus Infections", "incubation period", "transmission", "clinical characteristics", "physiopathology", "hyperglycemia", with the interposition of the Boolean operator" and "'." In addition, we access the scientific literature and recommendations currently available on WHO websites and the U.S. Centers for Disease Control and Prevention (CDC). Patients infected with SARS-CoV-2 and hyperglycemia (blood glucose $>180 \mathrm{mg} / \mathrm{dL}$ ) had higher mortality compared to patients with controlled blood glucose. Acute or chronic hyperglycemia and, moreover, when it

I. Médico internista. Hospital Essalud I Florencia de Mora. Profesor asociado, Departamento de Ciencias Básicas Médicas, Facultad de Medicina, Universidad Nacional de Trujillo, Trujillo, Perú.

2. Alumno de la Facultad de Medicina, Universidad Nacional de Trujillo, Trujillo, Perú. remains uncontrolled is associated with increased severity and mortality in patients infected with SARS-CoV-2.

Keywords: Hyperglycemia, severe acute respiratory syndrome, viral pneumonia, viral, coronavirus infections, COVID- 19.

\section{INTRODUCCIÓN}

El SARS-CoV 2, el nuevo coronavirus de China que apareció en diciembre del 2019 es causante de una nueva enfermedad llamada COVID-19(1), esta incluye cuadros respiratorios que varían desde síntomas respiratorios altos hasta cuadros de neumonía grave ${ }^{(2)}$. La transmisión de persona a persona la convierte en pandemia ${ }^{(3)}$.

En informes preliminares, presentan características clínicas de pacientes con la nueva infección por Covid-19, se observó hiperglucemia en el $51 \%$ de los $\operatorname{casos}^{(4)}$. Una hiperglucemia puede conducir a múltiples complicaciones, incrementando la gravedad de la infección y la tasa de mortalidad a comparación de individuos con glucemia bien controlada $^{(5)}$.

Este trabajo busca indagar si la hiperglucemia puede ser un factor pronóstico de mortalidad en pacientes con SARS- 
$\mathrm{CoV} 2$ y se procura dar una posible explicación mediante mecanismos fisiopatológicos. Además de vislumbrar nuevos conocimientos sobre este virus.

\section{MATERIAL Y MÉTODOS}

Se realizó una revisión de la literatura para buscar artículos en las bases de datos de PubMed y Google Scholar hasta el 02 de Junio del 2020. Se utilizó las palabras claves: "SARSCoV-2", "COVID-19", "infección", "patogénesis", " período de incubación "," transmisión "," características clínicas “, "fisiopatología”, "hiperglucemia”, " diabetes “, con la interposición del operador booleano" Y “. Además, se accedió a la literatura científica y las recomendaciones actualmente disponibles en los sitios web de la organización mundial de la salud (OMS), Asociación Americana de Diabetes (ADA) y los Centros para el Control y la Prevención de Enfermedades (CDC).

\section{HIPERGLUCEMIA \\ Definiciones}

Es una glucemia $>125 \mathrm{mg} / \mathrm{dL}$ durante el ayuno y $>180$ mg / dL dos horas postprandial.(6) La ADA define a la hiperglucemia de estrés como valores de glucemias $>140$ $\mathrm{mg} / \mathrm{dL}$ en pacientes hospitalizados sin diagnóstico previo de diabetes mellitus (DM). ${ }^{(7)}$ Un estudio retrospectivo consideró dos o más glucemias basales $>180 \mathrm{mg} / \mathrm{dL}$ dentro de 24 horas con una hemoglobina glucosilada $(\mathrm{A} 1 \mathrm{C})<6,5 \%$ o desconocida, y la definió como hiperglucemia no controlada. (8)

La hiperglucemia puede ser crónica y aguda. ${ }^{(9)}$ La primera caracteriza a la DM tipo 1, se presenta por la destrucción de las células beta $(\beta)$ del páncreas, generando una deficiencia absoluta de insulina. La DM tipo 2 es caracterizada por un defecto relativo de la insulina o aumento de la resistencia de su acción. ${ }^{(10)}$ La segunda es transitoria e incluye a la hiperglucemia por estrés, característico del paciente crítico no diabético, aunque puede presentarse en diabéticos. (11) Y, puede ser causada por un estrés orgánico (accidente cerebrovascular, sepsis, quemadura, intervención quirúrgica). (8)

\section{Tasa de letalidad en el paciente crítico}

La hiperglucemia diagnosticada en el momento de la admisión a Unidades de Cuidado Intensivo (UCI) se asoció con una tasa de mortalidad de $16 \%$ en comparación con un $3 \%$ para pacientes con diabetes previa. Esto sugirió que la hiperglucemia durante la admisión a UCI tenía mayor impacto de mortalidad en los pacientes sin diabetes comparado con los que ya tenían. ${ }^{(12)}$

\section{Fisiopatología}

Los mecanismos de este desorden varían dependiendo de la tolerancia a la glucosa y la capacidad de producción de insulina del paciente, tipo y severidad de la enfermedad, además fármacos empleados en el tratamiento. La hiperglucemia es frecuentemente la manifestación de una enfermedad crítica, resultado de cambios metabólicos y hormonales agudos tras una respuesta a la lesión y al estrés. ${ }^{(13)}$ La liberación de hormonas contrarreguladoras (catecolaminas, cortisol, hormona del crecimiento y glucagón) inducen resistencia a la insulina, incremento en la producción hepática de glucosa, deterioro en la utilización periférica de la glucosa y deficiencia relativa de insulina. ${ }^{(7,13)}$ La hiperglucemia induce la transcripción de factores proinflamatorios, como el factor nuclear $\kappa \beta(\mathrm{NF} \kappa \beta)$ y el activador de la proteína-1 de unión. La inducción de estos está asociado al aumento de expresión de genes codificadores de proteínas que pueden mediar la inflamación, agregación plaquetaria, apoptosis y disfunción endotelial. ${ }^{(7,13)}$ También asociado con un incremento en la generación de especies reactivas de oxígeno que pueden dañar a lípidos, proteínas y al ADN. La producción de superóxido y su reacción con el óxido nítrico en estas condiciones resulta en la producción de peroxinitrito, nitración de proteínas, y activación del $N F \kappa \beta$ si no se restablece la glucemia. ${ }^{(7)}$

\section{Objetivos glucémicos en enfermedad crítica}

En un ensayo clínico se demostró que el rango glucémico de $80-110 \mathrm{mg} / \mathrm{dL}$ redujo la mortalidad en un $40 \%$ en comparación con un rango glucémico de 180-215 mg/dL en pacientes críticos. Sin embargo, un estudio multicéntrico, con pacientes críticos asignados al azar al control glucémico intensivo (80-110 mg/dL) no tuvo ventaja significativa en comparación con objetivos glucémicos moderados (140$180 \mathrm{mg} / \mathrm{dL}$ ). El grupo de tratamiento intensivo tuvo tasas de hipoglucemia $(<70 \mathrm{mg} / \mathrm{dL})$ de 10 a 15 veces mayores causando mayor mortalidad. ${ }^{(14)}$ Por tanto, un objetivo de glucosa de 140 a $180 \mathrm{mg} / \mathrm{dL}$ es razonable en la mayoría de los pacientes críticos y no críticos. ${ }^{(14,15)}$

\section{SÍNDROME RESPIRATORIO GRAVE (SARS-COV-2): Patogénesis}

El SARS-CoV-2 que ingresa al organismo se dirige a los alvéolos, en un proceso de múltiples pasos que involucra. varios dominios distintos en la proteína espiga, que median su unión a la superficie celular, el compromiso del receptor, procesamiento de la proteasa y fusión de membranas. ${ }^{(16)} \mathrm{La}$ proteína S del SARS-CoV-2 se une a ACE2 a través de su subunidad S1. Luego es escindido por TMPRSS2 en el límite S1 / S2 o dentro de la subunidad S2, esto permite liberar el péptido de fusión interno con el dominio Spike TM, para la fusión de membranas virales y celulares, ocasionando que el genoma de ARN se libere en el citoplasma de la célula huésped. ${ }^{(17)}$ Aquí se une a ribosomas y a una polimerasa dependiente del ARN viral, produciendo copias íntegras o parciales de ARN, formando nuevos virus..$^{(15,17)}$

\section{Mortalidad por infección y gravedad}

La tasa de mortalidad de COVID-19 para China se informó inicialmente de 5,6\% (IC 95\%: 5,4-5,8\%). Para el 20 de febrero, la tasa bruta se estimó en 3,8\%.(18) Según cálculos actuales de la OMS basados en datos de Wuhan, el COVID-19 el 80\% de los casos cursa de forma leve o moderada, $15 \%$ graves, $5 \%$ cuidados intensivos y $4 \%$ mueren. Este porcentaje de muertes se repitió en países de 
Europa y Oceanía. A diferencia de los países de Sudamérica, EE.UU, México, Canadá, India y África, presentaronn un $10 \%$ de muertes. ${ }^{(19)}$ Esta pandemia puede causar cerca de 500 millones de muertes; es decir, el 6\% de la población mundial $\mathrm{y}$, potencialmente, más. ${ }^{(19)}$

\section{HIPERGLUCEMIA EN PACIENTES COVID-19 Tasa de letalidad}

En un estudio observacional retrospectivo con 1122 pacientes adultos COVID-19 confirmados en 88 hospitales de EE. UU, se halló que de los 184 pacientes con diabetes y/o hiperglucemia que murieron o fueron dados de alta, 40 de 96 pacientes con hiperglucemia no controlada $(41,7 \%)$ fallecieron en comparación con 13 de 88 pacientes con diabetes $(14,8 \%){ }^{(8)}$

\section{Fisiopatología}

Existen al menos dos razones por las cuales la hiperglucemia particularmente una aguda, puede ser muy peligrosa durante la infección por SARS-CoV-2. ${ }^{(20)}$ La primera es que la hiperglucemia aguda se acompaña de un gran aumento de mediadores inflamatorios conocido como "tormenta de citoquinas". ${ }^{(20)}$ El protagonista de esta tormenta es la interleucina 6 (IL-6), la cual actúa sobre una gran cantidad de células y tejidos, incluyendo el tejido pulmonar ${ }^{(21)}$. La segunda parece ser muy específica para SARS-CoV-2 y está relacionada a su unión a ACE2 en el neumocito tipo 2, mediante su glicoproteína S. ${ }^{(20)}$ En este punto la hiperglucemia genera una glucosilación alta y aberrante de ACE2 en el tejido pulmonar que podría aumentar los sitios de unión viral. ${ }^{(22)}$ Sumado a la depresión de la inmunidad innata que se ve comprometida en pacientes con hiperglucemia aguda y crónica, se da una fácil proliferación del patógeno dentro del huésped, permitiendo una mayor propensión a la infección y gravedad de la enfermedad. ${ }^{(23)}$ Se ha demostrado una gran tinción de anticuerpos para ACE2 en islotes pancreáticos, evidenciando que también puede expresarse en las células $\beta$ pancreáticas. ${ }^{(24)}$ Además, se ha reportado lesión pancreática aguda (amilasa y lipasa elevadas) en el $17 \%$ de los pacientes, sin identificar factor causal de pancreatitis aguda y los resultados de laboratorio mostraron linfopenia, aumento de marcadores inflamatorios (PCR, IL-6) y glucemia, relacionándolos con el empeoramiento del estado de salud. ${ }^{(25)}$

La actividad intrínseca del sistema local reninaangiotensina regula el flujo sanguíneo dentro del islote pancreático, lo que permite el reconocimiento de niveles elevados de glucosa y la oportuna liberación de insulina. Se ha evidenciado en roedores que el eje (ACE2)/ angiotensina (1-7)/receptor Mas protege a las células $\beta$ al mejorar la función de las células endoteliales microvasculares de los islotes. Además, ACE2 es un regulador protector negativo del sistema clásico de renina-angiotensina. ${ }^{(26)}$ La inhibición competitiva de la ACE2 por el virus SARS-2, reduce sus efectos protectores y por otra parte la angiotensina II intacta actúa a través del receptor de angiotensina 1 para ejercer respuestas proinflamatorias, así como la formación de radicales libres de oxígeno en los vasos sanguíneos por activación de las oxidasas de nicotinamida adenina dinucleótido, reducido y fosfato reducido que se encuentran aumentadas en estados de hiperglucemia. ${ }^{(27,28)}$ Estos cambios pueden explicar el retraso de liberación de insulina en respuesta a la glucosa, lo que lleva a una hiperglucemia aguda, como lo presentaron pacientes sin diabetes previa, en el transcurso de la hospitalización por la infección viral. ${ }^{(29)}$

\section{Importancia del control hiperglucémico en COVID-19}

Estudios descriptivos sugieren que muchos pacientes COVID-19 tienen hiperglucemia. Esto puede deberse a altos niveles de estrés, inflamación, falta de protocolos adecuados para el manejo de la glucosa y cambios en la dieta. ${ }^{(15)}$ Se ha reportado el uso de esteroides en hasta un $45 \%$ de los casos especialmente en pacientes graves, lo que puede ocasionar hiperglucemia. Por eso será importante controlar los niveles de glucosa en pacientes críticos COVID-19 y posiblemente agregar insulina a la terapia. El objetivo de glucosa de 140 a $180 \mathrm{mg} / \mathrm{dL}$ sugiere el inicio de la terapia con insulina con una hiperglicemia $\geq 180$ $\mathrm{mg} / \mathrm{dl} .{ }^{(15)}$ Se ha sugerido que se debe lograr objetivos de glucosa en sangre $<180 \mathrm{mg} / \mathrm{dL}$ en los pacientes diabéticos con COVID-19, sin provocar hipoglucemia, en ausencia de evidencia de lo contrario. ${ }^{(30)}$

\section{CONCLUSIÓN}

La hiperglucemia, aguda o crónica y, más aún, cuando permanece no controlada se asocia con un aumento de la gravedad y la mortalidad en los pacientes infectados con SARS-CoV2.

\section{REFERENCIAS BIBLIOGRÁFICAS}

1. Roussel Y, Giraud-Gatineau A, Jimeno M, Rolain J, Zandotti C, Colson $\mathrm{P}$ et al. SARS-CoV-2: fear versus data. Int. J. Antimicrob Agents [Internet]. 2020 [consultado 3 May 2020]; 9 (41): 105947. Disponible en: https:/www.sciencedirect.com/science/article/pii/ S0924857920300972

2. Centro de Coordinación de Alertas y Emergencias Sanitarias. Manejo en urgencias del COVID-19 [Internet]. Mscbs.gob.es. 2020 [consultado 4 May 2020]. Disponible en: https://www.mscbs. gob.es/profesionales/saludPublica/ccayes/alertasActual/nCovChina/documentos/Manejo_urgencias_pacientes_con_COVID-19. pdf

3. Wang A, Zhao W, Xu Z, Gu J. Timely blood glucose management for the outbreak of 2019 novel coronavirus disease (COVID-19) is urgently needed. Diabetes Res Clin Pract [Internet]. 2020 [consultado 3 May 2020]; 162: 108118. Disponible en: https:/www. ncbi.nlm.nih.gov/pmc/articles/PMC7102524/

4. Ilias I, Zabuliene L. Hyperglycemia and the novel Covid-19 infection: Possible pathophysiologic mechanisms. Medical Hypotheses [Internet]. 2020 [consultado 3 May 2020]; 139: 109699. Disponible en: https://www.sciencedirect.com/science/article/pii/ S0306987720303947

5. Zhu L, She Z, Cheng X, Qin J, Zhang X, Cai J et al. Association of Blood Glucose Control and Outcomes in Patients with COVID-19 and Pre-existing Type 2 Diabetes. Cell Metab [Internet]. 2020 [consultado 20 May 2020];:1-10. Disponible en: https://www. sciencedirect.com/science/article/pii/S 1550413120302382 ? via\%3Dihub

6. Mouri M, Badireddy M. Hyperglycemia [Internet]. Treasure Island: 
StatPearls Publishing; 2020 [consultado 7 May 2020]. Disponible en: https:/www.ncbi.nlm.nih.gov/books/NBK430900/

7. Aleman L, Guerrero J. Hiperglicemia por sepsis: del mecanismo a la clínica [Internet]. Rev Med Chile. 2018 [consultado 5 May 2020]:502-510. Disponible en: https://scielo.conicyt.cl/scielo. php?script=sci_arttext\&pid=S0034-98872018000400502\&lng=es.

8. Bode B, Garrett V, Messler J, McFarland R, Crowe J, Booth R et al. Glycemic Characteristics and Clinical Outcomes of COVID-19 Patients Hospitalized in the United States. J Diabetes Sci Technol [Internet]. 2020 [consultado 7 May 2020]:1-9. Disponible en: https:// glytecsystems.com/wp-content/uploads/JDST-Glytec-CovidResearch.pdf

9. Fabregate R, Fabregate M, Fernández A, Guerri A, García M, Muro $\mathrm{M}$ et al. Fisiopatología y manejo de la hiperglucemia intrahospitalaria [Internet]. 1st ed. Buenos Aires: Díaz de Santos; 2012 [consultado 7 May 2020]. Disponible en: https://www.editdiazdesantos.com/ wwwdat/pdf/9788499690476.pdf

10. MINSA. Guía de Práctica Clínica para el Diagnóstico, Tratamiento y Control de la Diabetes Mellitus Tipo 2 en el Primer Nivel de Atención [Internet]. Bvs.minsa.gob.pe. 2016 [consultado 7 May 2020]. Disponible en: http://bvs.minsa.gob.pe/local/MINSA/3466. pdf

11. Pérez A, Pérez J, Manzano F. Control de la hiperglucemia de estrés. Estado actual. Nutr Clin Med [Internet]. 2019 [consultado 7 May 2020];13(1):1-18. Disponible en: http://www.aulamedica.es/ nutricionclinicamedicina/pdf/5070.pdf

12. Pérez A, Guillén A, Fraire I, Anica E, Briones J, Carrillo R. Actualidades en el control metabólico del paciente crítico: hiperglucemia, variabilidad de la glucosa, hipoglucemia e hipoglucemia relativa. Cirugía y Cirujanos [Internet]. 2017 [consultado 7 May 2020]; 85 (1): 93-100. Disponible en: https:// www.sciencedirect.com/science/article/pii/S0009741116301074

13. Gracia A, Cruz M, Madrigal E, Morales J, Vera O. Manejo de la hiperglucemia en pacientes hospitalizados. Rev Med Inst Mex Seguro Soc [Internet]. 2015 [consultado 8 May 2020];53(2):192-199. Disponible en: https://www.medigraphic.com/pdfs/imss/im-2015/ im152n.pdf

14. American Diabetes Association. Diabetes Care in the Hospital: Standards of Medical Care in Diabetes-2020. Diab Care [Internet]. 2019 [consultado 26 May 2020]; 43 (Suplemento 1): S193-S202. Disponible en: https://care.diabetesjournals.org/ content/43/Supplement 1/S193

15. Al-Jaghbeer M, Lansang M. Manejo de la hiperglucemia en el paciente crítico con COVID-19. Cleve Clin J Med [Internet]. 2020 [consultado 5 May 2020]: 1-2. Disponible en: https://www.ccjm. org/content/early/2020/05/12/ccjm.87a.ccc012

16. Letko M, Marzi A, Munster V. Functional assessment of cell entry and receptor usage for SARS-CoV-2 and other lineage B betacoronaviruses. Nat Microbiol [Internet]. 2020 [consultado 19 May 2020];5(4):562-569. Disponible en: https://www.nature. com/articles/s41564-020-0688-y

17. Meng T, Cao H, Zhang H, Kang Z, Xu D, Gong H et al. The insert sequence in SARS-CoV-2 enhances spike protein cleavage by TMPRSS.bioRxiv[Internet].2020[consultado4Jun2020]. Disponible en: https:/www.biorxiv.org/content/10.1101/2020.02.08.926006v3. full

18. Basu A. Estimating the infection fatality rate among symptomatic COVID-19 cases in the United States. Health Affairs [Internet]. 2020 [consultado 8 May 2020];39(7):1-6. Disponible en: https:// www.healthaffairs.org/doi/10.1377/hlthaff.2020.00455

19. Grech V. Unknown unknowns - COVID-19 and potential global mortality. Early Hum Dev [Internet]. 2020 [consultado 18 May 2020];144:105026. Disponible en: https:/www.sciencedirect.com/ science/article/pii/S0378378220301973?via\%3Dihub

20. Bornstein S, Rubino F, Khunti K, Mingrone G, Hopkins D, Birkenfeld A et al. Practical recommendations for the management of diabetes in patients with COVID-19. Lancet Diabetes Endo
[Internet]. 2020 [consultado 10 May 2020]:1-4. Disponible en: https://www.thelancet.com/pdfs/journals/landia/PIIS2213-8587 (20)30152-2.pdf

21. Cascella M, Rajnik M, Cuomo A, Dulebohn S, Di Napoli R. Features, Evaluation and Treatment Coronavirus (COVID-19) [Internet]. Treasure Islan: StatPearls Publishing; 2020 [consultado el 19 de May. de 2020]. Disponible en: https://www.ncbi.nlm.nih.gov/books/ NBK554776/

22. Brufsky A. Hyperglycemia, hydroxychloroquine, and the COVID-19 pandemic. J Med Virol [Internet]. 2020 [consultado 19 May 2020]:1-6. Disponible en: https://onlinelibrary.wiley.com/ doi/full/10.1002/jmv.25887?fbclid=IwAR3187fD4j1O0Ma7QU3K6dgZ-ztWPOEi2yoKqmCYhpKLAb8o_adjSmkNrU

23. Pal R, Bhansali A. COVID-19, diabetes mellitus and ACE2: The conundrum. Diabetes Res Clin Pract [Internet]. 2020 [consultado 18 May 2020];162:108132. Disponible en: https://www.ncbi.nlm.nih. gov/pmc/articles/PMC7118535/

24. Sardu C, Gambardella J, Morelli M, Wang X, Marfella R, Santulli G. Hypertension, Thrombosis, Kidney Failure, and Diabetes: Is COVID-19 an Endothelial Disease? A Comprehensive Evaluation of Clinical and Basic Evidence. J Clin Med [Internet]. 2020 [consultado 15 May 2020];9(5):1417. Disponible en: https://www. mdpi.com/2077-0383/9/5/1417/htm

25. Wang F, Wang H, Fan J, Zhang Y, Wang H, Zhao Q. Pancreatic injury patterns in patients with COVID-19 pneumonia. Gastroenterology [Internet]. 2020 [consultado el 31 de May. de 2020 ]. Disponible en: https://www.gastrojournal.org/article/S00165085(20)30409-1/pdf

26. Lu C, Wang Y, Yuan L, Li Y, Li X. The angiotensin-converting enzyme 2/angiotensin (1-7)/Mas axis protects the function of pancreatic $\beta$ cells by improving the function of islet microvascular endothelial cells. International Journal of Molecular Medicine [Internet]. 2014 [consultado 16 May 2020];34(5):1293-1300. Disponible en: https://www.spandidos-publications.com/10.3892/ ijmm.2014.1917

27. Astuti I, Ysrafil. Severe acute respiratory syndrome coronavirus 2 (SARS-CoV-2): An overview of viral structure and host response. Diabetes Metab Syndr [Internet]. 2020 [consultado 7 May 2020]; 14 (4): 407-412. Disponible en: https://www.sciencedirect.com/ science/article/pii/S1871402120300849\#bib12

28. Katulanda P, Dissanayake H, Ranathunga I, Ratnasamy V, Wijewickrama $\mathrm{P}$, Yogendranathan $\mathrm{N}$ et al. Prevention and management of COVID-19 among patients with diabetes: an appraisal of the literature. Diabetologia [Internet]. 2020 [consultado 17 May 2020];. Disponible en: https://link.springer.com/ article/10.1007/s00125-020-05164-x

29. Bornstein S, Dalan R, Hopkins D, Mingrone G, Boehm B. Endocrine and metabolic link to coronavirus infection. Nat Rev Endocrinol [Internet]. 2020 [consultado 18 May 2020];16(6):297-298. Disponible en: https://www.nature.com/articles/s41574-020-0353-9

30. Singh A, Singh R. Does poor glucose control increase the severity and mortality in patients with diabetes and COVID-19? Diabetes Metab Syndr [Internet]. 2020 [consultado 5 May 2020];14(5):725-727. Disponible en: https:/www.ncbi.nlm.nih.gov/ pmc/articles/PMC7251348/?fbclid=IwAR1 ohNrojsqAXjYFbuj9ty jL_QVMou6r5v9teAiZ5RQmZDVumyBjPPD8WH0\#bib11

Conflicto de interés: Ninguno según los autores.

Financiamiento. Por los autores.

\section{CORRESPONDENCIA}

Luis Arturo Camacho Saavedra

Icamacho@unitru.edu.pe, Icamacho_saavedra@hotmail.com

Fecha de recepción: 14-09-2020.

Fecha de aceptación: |6-|| -2020. 